Musées, Patrimoine et Culture scientifiques et techniques

$116 \mid 2008$

mars - avril 2008

\title{
Évaluation de la toxicité des spécimens naturalisés
}

\section{Amandine Péquignot}

URL : http://journals.openedition.org/ocim/367

DOI : $10.4000 /$ ocim.367

ISSN : 2108-646X

Éditeur

OCIM

Édition imprimée

Date de publication : 1 mars 2008

Pagination : 4-9

ISSN : 0994-1908

Référence électronique

Amandine Péquignot, «Évaluation de la toxicité des spécimens naturalisés », La Lettre de l'OCIM [En

ligne], 116 | 2008, mis en ligne le 01 janvier 2011, consulté le 19 avril 2019. URL : http://

journals.openedition.org/ocim/367 ; DOI : 10.4000/ocim.367 


\section{Évaluation de la toxicité des spécimens naturalisés}

\section{Amandine Péquignot*}

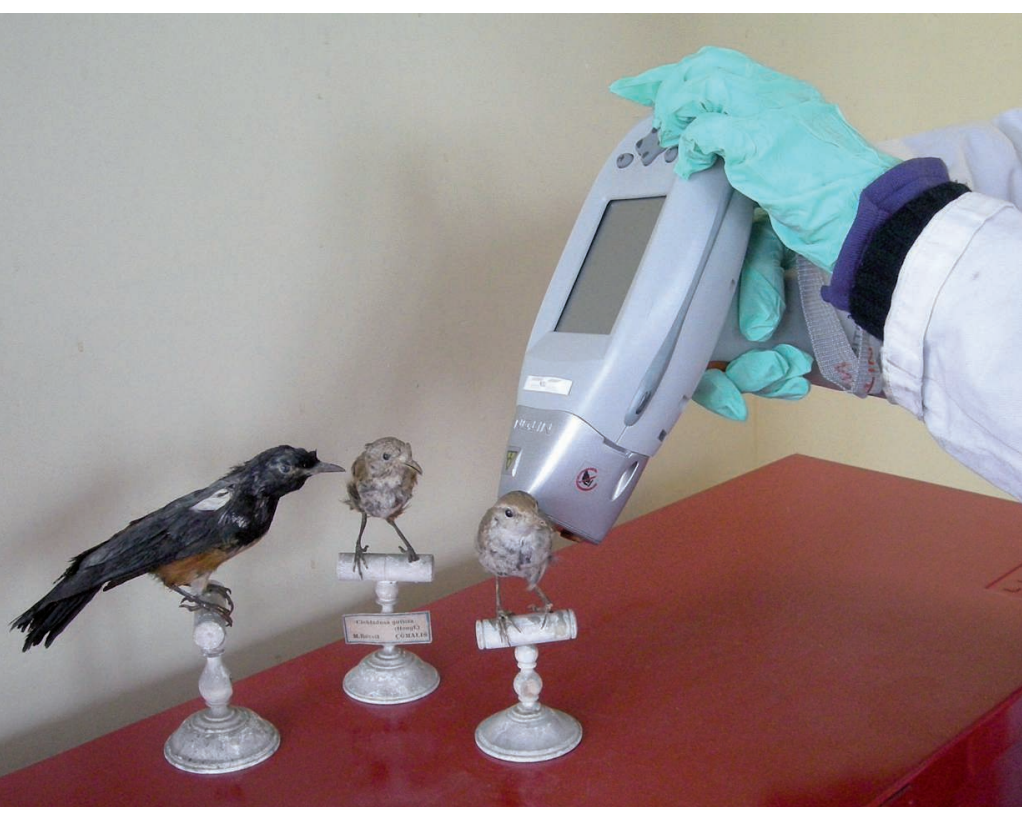

Utilisation du portable XRF Niton sur des oiseaux naturalisés (๑) A. Péquignot

\footnotetext{
* Amandine Péquignot est maître de conférences au centre de recherches sur la conservation
} des collections (MNHN-CNRS-MCC UMR 7188) au Muséum national d'Histoire naturelle apeq@mnhn.fr
L'expérience présentée ici consiste à appliquer la spectrométrie à fluorescence $X$ portable pour détecter la présence de résidus toxiques (arsenic, plomb, mercure) dans des collections d'oiseaux naturalisés. Cette technique d'analyse permet notamment de mettre en évidence la répartition inégale de ces éléments au sein des spécimens étudiés.

Grâce aux rares documents historiques et aux récentes publications, la question de la contamination des objets culturels par les pesticides n'étonne désormais plus la communauté muséale, mais elle demeure néanmoins délicate. En effet, le sujet soulève différentes interrogations quant aux problèmes de santé publique que cela implique pour les personnes travaillant au contact des spécimens mais également quant à la gestion et la pratique au quotidien des collections (consultation, stockage, nettoyage, restauration, prêt, exposition...). Les règles actuelles d'hygiène et de sécurité chargent les établissements et leurs institutions de tutelle d'informer précisément les personnels des dangers potentiels auxquels ils peuvent être confrontés, de fournir des moyens de protection et des lieux de travail adaptés, mais également de prendre leurs responsabilités en tant que lieu accueillant du public. Cette démarche implique avant toute chose de faire un état des lieux afin d'identifier et de quantifier clairement ces résidus toxiques présents au sein des différents types de collections.

Dans ce processus, la sélection des techniques d'analyse est un premier point crucial où rentrent en 
compte leur limite de détection, leur aspect destructif ou non, leur adéquation avec la nature de l'objet à analyser, la facilité et le coût de leur utilisation... Suite à nos premières investigations sur l'évaluation de spots tests pour la détection de l'arsenic (Marte et al., 2006), nous avions attiré l'attention sur l'importance de l'échantillonnage de l'objet pour obtenir des résultats significatifs.

Dans l'optique de mettre en place une méthodologie d'échantillonnage, nous avons étudié, dans le cas précis d'oiseaux naturalisés, la répartition des résidus inorganiques toxiques (arsenic, mercure et plomb) au moyen de la spectrométrie à fluorescence $\mathrm{X}$ portable afin d'identifier les zones les plus révélatrices.

\section{Principes et intérêts de I'XRF portable}

Pour détecter les résidus inorganiques potentiellement toxiques, comme par exemple l'arsenic (As), le mercure $(\mathrm{Hg})$ et le plomb $(\mathrm{Pb})$, il existe un large panel d'examens ou de techniques analytiques. Parmi celles-ci, la spectrométrie de fluorescence X (XRF) est une technique rapide, non-destructive, quantitative et qui a l'avantage d'être multi-élémentaire. Le principe de la fluorescence $X$ est une émission secondaire de rayons $\mathrm{X}$, caractéristique des éléments atomiques qui composent l'échantillon. L'échantillon à analyser est soumis à un faisceau de rayons $\mathrm{X}$ qui se présente selon les instruments sous différentes formes : un flux d'électrons, une source radioactive ou un tube à rayons $\mathrm{X}$. Sous l'effet des rayons $\mathrm{X}$, l'échantillon " entre en résonance ", éjectant les électrons des couches internes des différents atomes par effet photoélectrique. Ces lacunes électroniques ainsi formées sont comblées par des

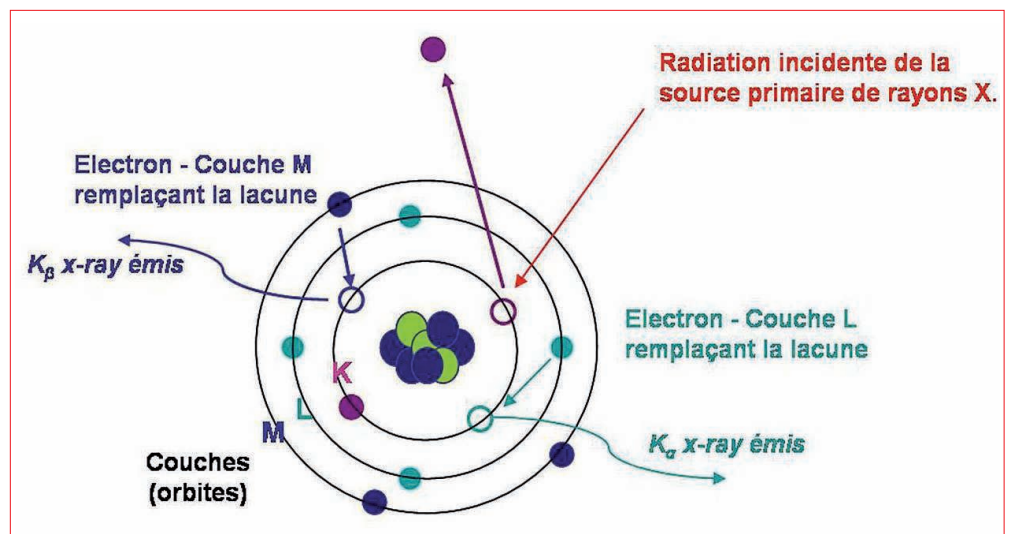

Principe de la fluorescence $X$ électrons des couches plus externes. Cette transition électronique s'accompagne de l'émission d'un rayonnement dit de fluorescence $\mathrm{X}$ dans le cas des photons comme source d'excitation, ou d'émission X dans le cas des particules chargées accélérées (électrons, protons). L'énergie du rayonnement $\mathrm{X}$ émis est caractéristique de l'atome excité, et correspond à la différence d'énergie de liaison entre les couches électroniques correspondant au niveau d'excitation (K, L, M...). La mesure des différentes énergies du rayonnement $\mathrm{X}$ émis par un échantillon permet d'identifier les numéros atomiques $\mathrm{Z}$ des différents éléments le constituant.

Dans le cas particulier de l'étude, la spectrométrie a été utilisée sous son aspect portable au moyen du Niton Xlt 700 où les rayons $X$ sont obtenus au moyen d'un tube à rayons $X^{(1)}$ constitué d'une source d'électrons (cathode) dont les caractéristiques sont les suivantes : tension maximale du tube $\mathrm{X}$ $35 \mathrm{kV}$, courant maximal $10 \mu \mathrm{A}$ et puissance maximale 1,7 W. La section du faisceau (spot) est approximativement de $20 \mathrm{~mm}$ de diamètre au contact de l'échantillon. En raison de son aspect portatif, la sensibilité de la spectrométrie est moindre que celle dite classique que l'on trouve en laboratoire. Les éléments pouvant être détectés sont de $\mathrm{Z}=24$ (chrome) à $\mathrm{Z}=92$ (uranium). Le niveau de détection varie selon le type d'éléments analysés, la matrice du matériau et le temps d'acquisition de l'analyse. L'instrument considère une erreur de lecture de $2 \sigma$ (déviation standard) pour les résultats. L'aspect non-destructif et, depuis peu, la portabilité de l'instrument, permettent des changements remarquables dans les critères d'utilisation de la fluorescence X pour l'étude des objets. Cela explique en grande partie ses applications dans le domaine du patrimoine culturel comme par exemple la caractérisation de pigments (Hochleitner et al., 2003), l'examen de certains éléments en archéométrie (Helmig et al., 1989) ou de divers objets culturels (Dussubieux et al., 2005). Depuis peu, l'XRF est employée plus spécifiquement à des fins de santé publique comme par exemple pour l'étude des risques liés à la présence de peintures au plomb dans les logements (Anonyme, 2005). Cette perspective trouve écho dans le domaine muséal où la détection des résidus toxiques fait l'objet d'une attention accrue en raison des problèmes moraux et juridiques qu'elle soulève. Des premières investigations ont été réalisées dans les collections ethnographiques (Boulton, A., 1986 ; Odegaard et al., 2005), les herbiers (Oyarzun, 2007) mais plus rarement dans les collections de spécimens naturalisés (Sirois, 2001). 


\section{Arsenic... et autres volatiles}

L'étude a porté sur 200 falconiformes conservés au Muséum national d'Histoire naturelle couvrant une large période (1806 à 2004). Ces oiseaux ont des origines diverses : 169 spécimens proviennent de la collection dite générale représentant à peu près 75 collecteurs différents, 18 appartiennent à la collection Anfrie (1880-1907) et enfin 13 font partie de la collection Marmottant (1888). Les spécimens étudiés sont stockés dans les réserves souterraines du Muséum (zoothèque) et représentent près de deux Compactus ${ }^{\circledR}$ (armoires mobiles) parmi ceux consacrés aux falconiformes. Seuls les spécimens de la collection Marmottant sont stockés dans d'autres Compactus ${ }^{\circledR}$ de la salle ornithologique.

Hormis les méthodes de préparation utilisées pour les spécimens récemment naturalisés au Muséum, aucune information n'a été réunie concernant celles employées jadis pour les différentes collections, le lieu et l'auteur de la naturalisation, et les traitements insecticides qu'auraient pu subir les spécimens avant ou depuis leur intégration aux collections nationales.

La méthode d'échantillonnage mise en place a été testée et évaluée auparavant sur des oiseaux naturalisés réformés, et a pris en compte différents paramètres jugés essentiels dans la détection des produits toxiques et leur suivi au sein de la collection.

Tout abord, l'environnement de l'objet. Son emplacement par rapport aux autres spécimens, et sa localisation au sein du Compactus ${ }^{\circledR}$ (étagère, tiroir...) sont notés afin d'assurer une continuité géographique des analyses. Des relevés au niveau des étagères, du sol et des murs sont également effectués de façon aléatoire au cours de l'étude.

Ensuite, l'objet lui-même. Chaque oiseau est analysé selon une série constante de 17 localisations couvrant au mieux l'ensemble du spécimen, à savoir l'oiseau naturalisé et son socle. Après chaque analyse, la fenêtre du détecteur est soigneusement nettoyée avec de l'éthanol $95 \%$ pour éliminer toute éventuelle accumulation de poussière qui, au final, biaiserait les résultats. Une calibration de l'instrument est pratiquée après 120 analyses, ce qui correspond à 7 oiseaux étudiés, généralement en une demi-journée.

Le temps d'acquisition pour chaque spot a été établi à partir d'analyses faites sur des standards EPA Reference Method (SW486-RCRA) dont la concentration des éléments est connue, tout en augmentant le temps d'analyse $(2,10,20,40,60,90,120$ et 180 secondes $)$ et en maintenant linstrument immobile dans une plate-forme. Le temps retenu de 80 secondes répond à la fois à une détection correspondant à la concentration $(+/-2 \sigma)$ des éléments (essentiellement $\mathrm{As}, \mathrm{Hg}, \mathrm{Pb}$ ) présents dans le standard testé. Bien sûr, le temps d'analyse peut être augmenté pour une meilleure définition, mais un des intérêts de I'XRF portable est de fournir des résultats qualitatifs et semi-quantitatifs avec une certaine rapidité
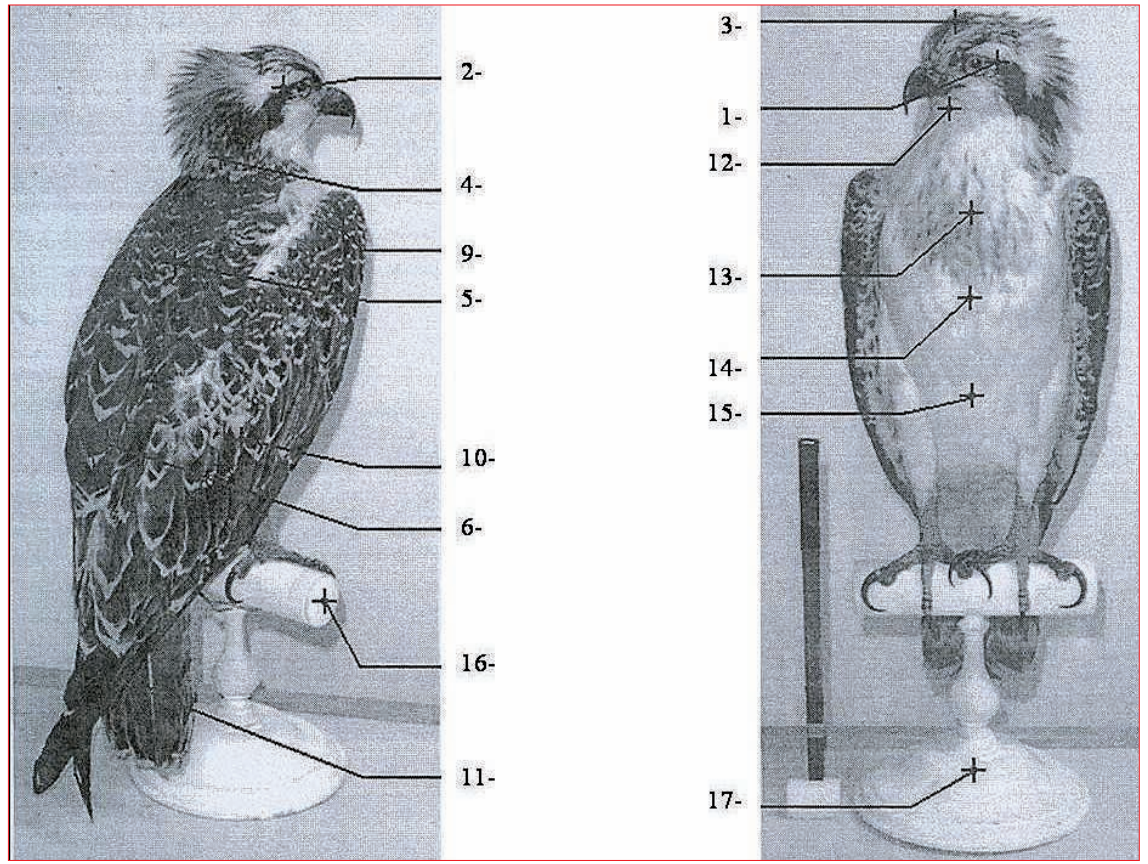
sur le terrain ! La méthodologie mise en place tente de concilier au mieux le temps total consacré à chaque spécimen (25-30 minutes) et des résultats significatifs.

\section{Des résultats et beaucoup de discussions}

Les résultats obtenus montrent que $100 \%$ des spécimens analysés sont positifs à l'arsenic indépendamment du collecteur et de l'époque de préparation. Une majorité (62\%) contient un niveau d'arsenic élevé (10 000-49 999 ppm) ${ }^{(2)}$, et $33 \%$ un niveau jugé moyen (1 000-9 999 ppm). En revanche, très peu d'oiseaux (1\%) ont un niveau très élevé (50 000-200 000 ppm). Les spécimens présentant les plus faibles concentrations de «traces » sont ceux récemment préparés au MNHN (1989-1997-2004),

Répartition des 17 points d'analyse sur le spécimen (c) A. Péquignot auxquels on peut associer les 5 spécimens datant du début du XXe siècle et dont le niveau est jugé « bas ». 
$\lceil$ As\ par spécimen $=$ moyenne de 15 relevés effectués sur chaque oiseau - ne comprenant pas le socle-

TOTAL : 200 spécimens étudiés

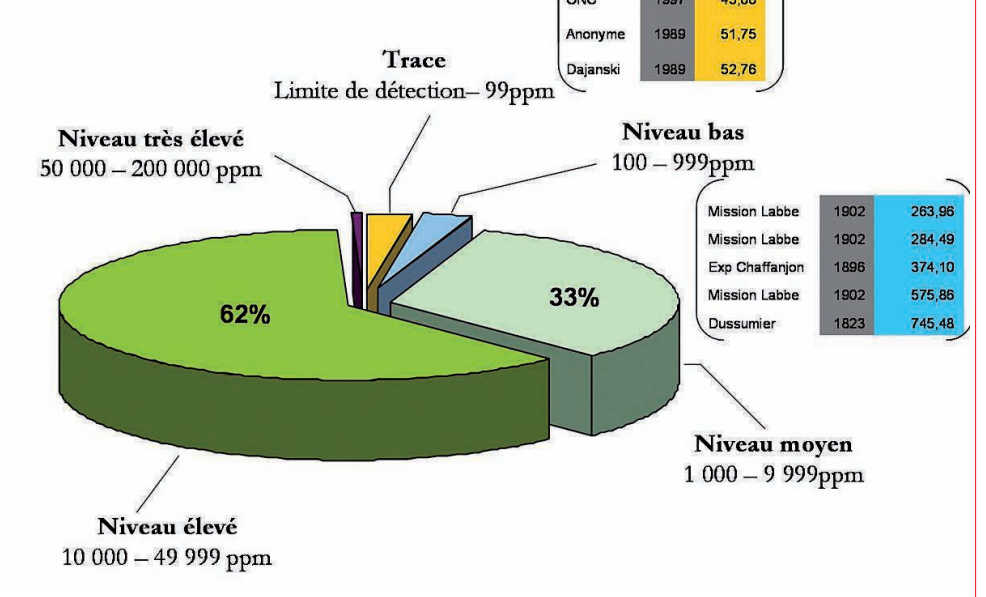

Résultats concernant les niveaux d'arsenic sur les 200 spécimens analysés, prenant en compte la moyenne par objet des 15 relevés effectués sur l'oiseau naturalisé.

Grâce à la série d'analyses de 17 points constants sur l'ensemble de l'objet, il est possible d'étudier la répartition de l'élément au sein de l'objet et de dégager un profil arsenical qui laisse apparaître des zones à forte concentration. Ainsi, l'arsenic se localise essentiellement au niveau de l'oiseau lui-même, avec une prédominance au niveau de la tête et du corps (poitrine, dos). Par ailleurs, des quantités inégales sont observées au niveau des ailes : les parties supérieures étant plus arsenifiées que les extrémités. Cette distribution concorde assez précisément avec le mode opératoire du tannage au savon arsenical décrit dans les manuels de taxidermie aux $\mathrm{XIX}^{\mathrm{e}}$ et $\mathrm{XX}^{\mathrm{e}}$ siècles (Péquignot, 2001).

Ce profil arsenical a été observé pour tous les spécimens, exceptés ceux récemment naturalisés. Pour ces derniers, la répartition de l'arsenic est homogène et en faible quantité sur toute la surface de l'objet. Ces résultats soulèvent une interrogation : comment ces spécimens récents peuvent être positifs à l'arsenic après trois à huit ans de stockage dans la zoothèque? Différentes hypothèses peuvent expliquer l'origine de cette présence régulière mais faible d'arsenic sur des spécimens contemporains. La première explication serait qu'un traitement pesticide à base d'arsenic a été appliqué dans les réserves. Or la zoothèque n'a pas fait l'objet de ce type de traitement depuis sa création (1986). La seconde est celle de la contamination des spécimens qui peut être liée au système d'aération présent dans la zoothèque, à la gestion des spécimens (manipulation et déménagement de spécimens « contaminés » autour de spécimens « sains »), et à l'ouverture répétitive des Compactus $\AA$ créant des appels d'air importants. Les analyses des tiroirs des Compactus $\AA$, ainsi que les murs et les sols des locaux où sont stockés les falconiformes montrent la présence de poussière arsenicale dans les mêmes proportions que sur les spécimens récemment naturalisés. Ces résultats confirment fortement notre hypothèse de contamination, et font par ailleurs écho aux conclusions de Schieweck et al. (2007) sur la contamination de l'environnement des réserves par l'arsenic dans les muséums.

Contrairement à l'arsenic, les concentrations les plus importantes de plomb sont localisées au niveau des socles avec en moyenne : 5700 ppm (collection Marmottant), 39200 ppm (collection Marmottant) et 250000 ppm (collection générale). L’origine de ce taux excessivement élevé de plomb est à mettre vraisemblablement en relation avec la couleur blanche classique des socles des muséums. En effet, le pigment appelé «blanc de plomb » $\left(2 \mathrm{PbCO}_{3} \bullet \mathrm{Pb}(\mathrm{OH})_{2}\right)$ était souvent la base des peintures blanches utilisées aux $\mathrm{XIX}^{\mathrm{e}}-\mathrm{XX}^{\mathrm{e}}$ siècles (Feller, 1986). Par ailleurs, le plomb a également été détecté au niveau de l'oiseau même. Cela serait dû à l'usage de traitements insecticides à base d'arsenate de plomb $\left(\mathrm{AsO}_{8} \mathrm{~Pb}_{3}\right)$ communément utilisés

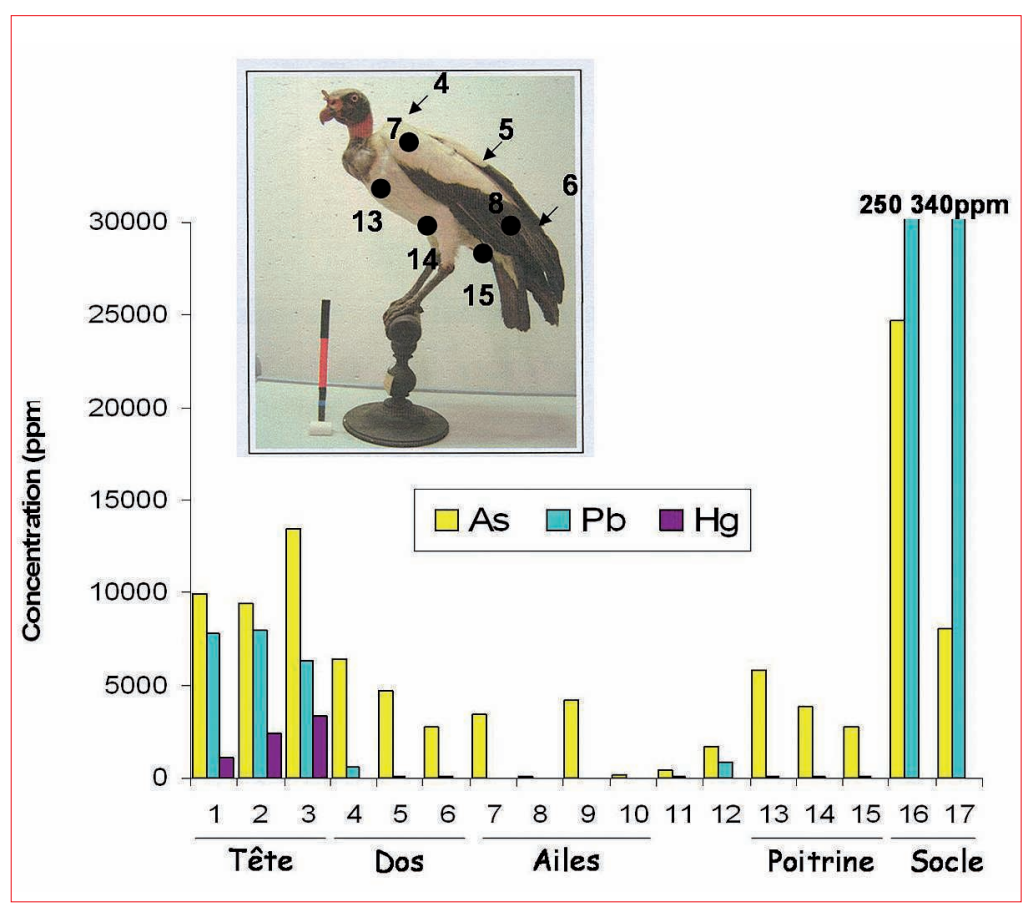

Exemple de la répartition et de la concentration de l'arsenic de Gypagus papa (Bonaparte, CG n ${ }^{\circ}$ 806-132) 
dans les collections au XIX ${ }^{\mathrm{e}}$ siècle ou à la contamination par la poussière de plomb provenant des socles. Mais ces hypothèses restent à confirmer.

Enfin, les analyses établissent la présence de mercure en concentration assez variable selon les collections : $89 \%$ de la collection générale est positive au mercure avec une moyenne 1000 ppm principalement localisée au niveau de la tête (autour des yeux), $75 \%$ de la collection Anfrie (300 ppm) et seulement $1 \%$ de la collection Marmottant (20 ppm). Cependant, la présence de mercure est selon nous à mettre davantage en relation avec l'espèce ornithologique et sa coloration « naturelle ». En effet, lorsqu'il présente une forme de concentration élevée, le mercure est essentiellement présent dans les spécimens colorés arborant des teintes rouges et/ou orangées, ce qui laisserait penser à l'utilisation du pigment vermillon (sulfure de mercure, $\mathrm{HgS}$ ). Par ailleurs, la présence d'arsenic dans les mêmes zones de couleurs peut être liée aux pigments rouge et jaune arsenifiés (réalgar, $\mathrm{As}_{4} \mathrm{~S}_{4}$ et l'orpiment, $\mathrm{As}_{2} \mathrm{~S}_{3}$ ). L'XRF portable ne pouvant détecter correctement les éléments faibles comme le soufre $(Z=16)$ ou le chlore $(Z=17)$, une analyse en diffraction X (XRD) permettrait d'éclaircir l'origine du mercure et de l'arsenic dans ces zones colorées.

\section{Conclusions}

Cette étude portant sur 200 oiseaux naturalisés a permis de tester la spectrométrie à fluorescence $\mathrm{X}$ portable, une technique d'analyse très innovante et ayant des applications depuis quelques années dans le domaine de la conservation du patrimoine culturel. Cette technique répond très favorablement aux objectifs premiers de l'étude, à savoir d'obtenir des réponses significatives et rapides quant à la présence ou l'absence de résidus potentiellement toxiques sur des objets. Par ailleurs comme tout instrument dit « nomade », l'XRF portable permet d'intervenir directement dans les collections, qu'elles soient exposées dans les vitrines ou stockées dans les réserves, limitant ainsi le déplacement des objets souvent non souhaitable et/ou parfois infaisable pour des raisons d'état de conservation de l'objet ou de taille. À l'instar de nombreux instruments nomades, l'XRF portable offre des résultats semi-quantitatifs permettant néanmoins d'avoir un ordre de grandeur quant à la concentration des éléments détectés. La précision de ces résultats reste cependant liée à l'effet matriciel de l'objet, c'est-à-dire aux éventuelles déviations que peuvent subir les rayons $\mathrm{X}$ au niveau

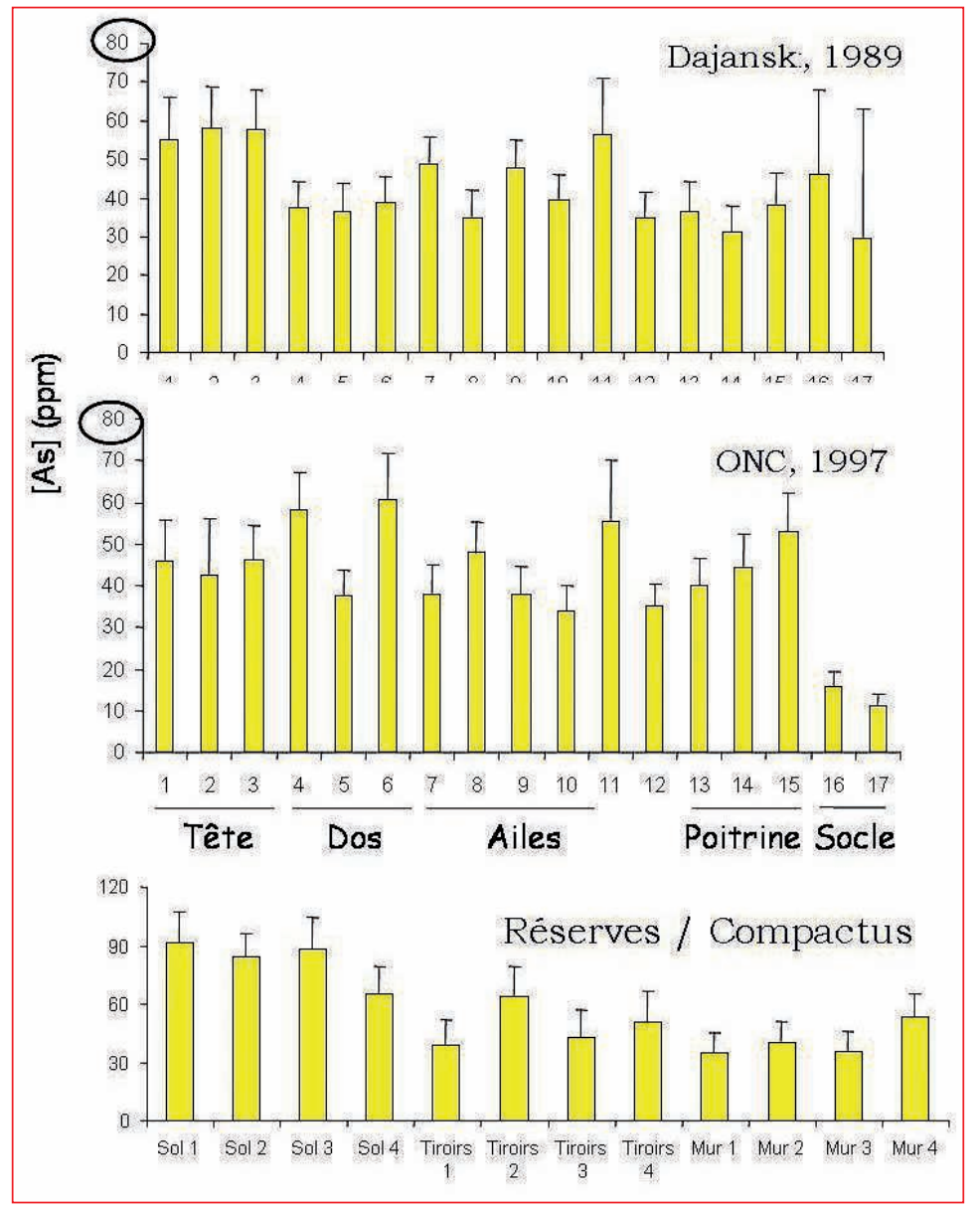

Profils arsenicaux de deux Accipiter gentilis (Dajanski, CG n 1989-242 / ONC, CG n 1997-1275)

et les concentrations d'arsenic relevées à différents lieux de la zoothèque (tiroirs, sol et mur) où sont stockés les falconiformes.

de l'incidence et de la réflexion, au sein des deux matrices physiquement et chimiquement très différentes (à savoir la peau et les plumes dans le cas précis). L'influence de cet effet matriciel est actuellement en cours d'étude.

Les résultats obtenus démontrent que la totalité des spécimens étudiés sont positifs à l'arsenic, au plomb ou au mercure avec des valeurs différentes selon des localisations bien spécifiques, indépendamment de la collection et de l'époque de la naturalisation. La méthodologie d'analyse mise au point a permis de mettre en évidence cette répartition inégale des éléments au sein même de l'objet, et de préconiser ainsi des zones prioritaires d'échantillonnage (tête, dos, ventre) afin d'obtenir des résultats significatifs dans le cadre de tests simples d'identification comme les spot tests. Néanmoins, nous appelons à la vigilance quant à l'interprétation des résultats prouvant la présence des éléments toxiques. En effet, une nuance 
doit être introduite au regard de la nature et de l'origine de ces éléments. Pour prendre l'exemple de l'arsenic, cet élément a des origines diverses (tannage, traitements insecticides, pigment...), des applications et des localisations variées au sein du spécimen (savon/pommade étalée à l'intérieur de la peau, liqueur ou solution appliquée uniquement sur les phanères, immersion de la peau dans un bain, saupoudrage insecticide des spécimens naturalisés...) et par conséquent une nature chimique bien différente (trioxide, sulfuré, plomb...). Cette nature chimique de l'élément, la nature matricielle de l'objet (peau, plume, bois...), le temps et les paramètres de conservation vont influer sur le comportement et la libération de l'élément potentiellement toxique en surface.

Pour l'interprétation de certains résultats, nous avons été confrontés au problème de la contamination des spécimens récents et "sains » par des poussières, notamment arsenicales. Cet aspect, ainsi que les résultats provenant des analyses des locaux et des cabinets de stockage soulèvent des questions quant à la gestion et à la pratique des collections : l'incorporation de nouveaux objets « sains » pouvant être contaminés à terme, l'accessibilité des locaux et la protection du personnel, le nettoyage ou dépoussiérage de ces spécimens dans des locaux souvent non-adaptés, le prêt de collections à des établissements qui pourraient se voir contaminés de ce fait... Quant à l'exposition des collections au niveau du public dans les musées... le débat est ouvert !

Enfin, nous attirons l'attention sur le fait que les pesticides et les biocides ne posent pas uniquement des problèmes de santé publique dans les musées. Bien que leur emploi soit assez efficace pour la préservation des collections, ils se trouvent désormais être les responsables de dégradations irréversibles sur les spécimens traités, comme en témoignent les travaux de Pohland et Mullen (2006) démontrant leurs effets décolorants sur le plumage des oiseaux naturalisés. Ceci questionne les limites de l'utilité de ces collections traitées et visiblement altérées pour la communauté scientifique. À vouloir parfois trop conserver...

Nous tenons à remercier le Professeur Michel Tranier, ancien directeur des collections du Muséum national d'Histoire naturelle qui a permis cette étude, et Noémie Hadjadj qui a réalisé dans le cadre d'un stage une étude préliminaire pour ces travaux.

\section{Notes}

(1) Un spectromètre XRF portable comportant une source radioactive est interdit en France.

(2) Nous reprenons les niveaux et les valeurs correspondants (en ppm) de Bengton, L. (2005).

\section{Bibliographie}

Anonyme, Rapport-Détection du plomb dans les peintures anciennes, Agence française de sécurité sanitaire environnementale (AFSSE), juin, 2005, 67 p.

Bengton, L. Testing for pesticide residues in the public program collections at the Royal B.C. Museum, ICOM-CC Ethnographic Conservation Newsletter, $n^{\circ} 26,2005$, pp. 7-8.

Boulton, A. The Examination, Treatment and Analysis of a Pair of Boots from the Aleutian Islands including a Note about Possible Pesticide Contamination, Journal of the American Institute for Conservation, $\mathrm{n}^{\circ} 25$ (1), 1986, pp. 1-13.

Dussubieux, L., Pinchin, S.-E., Tsang, J-S. et Tumosa, Ch.-S. Non-destructive elemental analysis : reliability of a portable X-ray fluorescence spectrometer for museum applications, in Preprints of the 14th Triennial Meeting ICOM Committee for Conservation, The Hague, 12-16 September 2005, 2005, pp. 766-773.

Feller R.-L. Ed. Artists' pigments : A handbook of their history and characteristics, Vol. 1, Washington : National Gallery of Art.; Vol. 2, Ashok Roy, editor ; Vol. 3, Elisabeth West FitzHugh, editor; Vol. 4, Barbara H. Berrie, 1986-2007.

Helmig, D., Jackwerth, E. et Hautmann, A. Archaeometallurgical fieldwork and the use of a portable X-ray spectrometer, Archaeometry, n³1 (2), 1989, pp. 181-191.

Hochleitner, B., Desnica V., Mantle M. et Schreiner, M. Historical pigments : a collection analyzed with X-ray diffraction analysis and X-ray fluorescence analysis in order to create a database, Spectrochimica Acta Part B : Atomic Spectroscopy, n58 (4), 2003, pp. 641-649.

Marte, F., Péquignot, A. et Von Endt, D. Arsenic in Taxidermy collection : history, detection, and management, Collection Forum, n²1 (1-2), 2006, pp. 143-150.

Odegaard, N. et Sadongei., A. Old Poissons, New Problems, a Museum Resource for Managing Contaminated Cultural Materials, New York AtlaMira Press, 2005, $126 \mathrm{p}$

Oyarzun, R., Higueras, P., Esbrí, J.-M. et Pizarro, J. Mercury in air and plant specimens in herbaria : a pilot study at the MAF Herbarium in Madrid (Spain), Science of the Total Environment, $\mathrm{n}^{\circ} 387,2007$, pp. 346-352.

Péquignot, A. Histoire de la Taxidermie en France de 1729-1928 : étude des facteurs de ses évolutions techniques et conceptuelles, et ses relations à la mise en exposition du spécimen naturalisé. Thèse de Doctorat, Paris : Muséum national d'Histoire naturelle, 2002, 390 p.

Pohland, G. et Mullen, P. Perservation agents influence UV-coloration of plumage in museum bird skins, Journal of Ornitholology, $n^{\circ} 147$ (3), 2006, pp. 464-467.

Schieweck, A., Delius W., Siwinski N., Vogtenrath, W., Genning, C. et Salthammer T. Occurrence of organic and inorganic biocides in the museum environment, Atmospheric Environment, n41, 2007, pp. 3266-3275.

Sirois, P.-J., The analysis of museum objects for the presence of arsenic and mercury : non-destructive analysis and sample analysis, Collection Forum, ${ }^{\circ} 16$ (1-2), 2001, pp. 65-75. 